\title{
Totally thoracoscopic closure of ventricular septal defect without a robotically assisted surgical system: A summary of 119 cases
}

\author{
Zeng-Shan Ma, MD, ${ }^{\text {a,b,c }}$ Chang-Yong Yang, MD, ${ }^{a}$ Ming-Feng Dong, MD, ${ }^{\mathrm{c}}$ Shu-Ming Wu, MD, ${ }^{\mathrm{a}}$ and \\ Le-Xin Wang, $\mathrm{MD}, \mathrm{PhD}^{\mathrm{b}, \mathrm{c}}$
}

Objectives: To summarize the clinical outcomes of totally thoracoscopic closure of a ventricular septal defect (VSD).

\begin{abstract}
Methods: Totally thoracoscopic VSD closure was performed in 119 patients (66 boys; mean age, $7.1 \pm 3.6$ years). An additional 35 patients undergoing open-chest VSD closure were selected as a control group. Using 3 port incisions in the right chest, pericardiotomy, bicaval occlusion, atriotomy, and VSD closure were performed by thoracoscopy without the aid of a robotically assisted surgical system.

Results: Cardiopulmonary bypass and aortic crossclamp times were $42.2 \pm 9.8$ and $32.5 \pm 7.3$ minutes, respectively. There were no deaths but 1 patient required insertion of a permanent pacemaker as a result of postoperative atrioventricular conduction block. The length of stay in the intensive care unit $(11.0 \pm 2.6$ vs $22.9 \pm 4.9$ hours, $P<.01)$ or postoperative hospital stay $(4.2 \pm 1.1$ vs $6.6 \pm 2.1$ days, $P<.03)$ in the thoracoscopic group were shorter than in the control group. The percentage of patients who required postoperative opioid analgesics in the thoracoscopic group was lower than in the control group $(31.9 \%$ vs $74.2 \%, P<.001)$. Rate of blood transfusion during the operation $(17.6 \%$ vs $65.7 \%, P=.001)$ and the postoperative use of opioid analgesics $(31.9 \%$ vs $74.3 \%, P=.003)$ in the thoracoscopic group was lower than in the control group. Transesophageal echocardiographic analysis $4.6 \pm 2.3$ months after the operation showed complete closure of the defect.
\end{abstract}

Conclusions: Totally thoracoscopic closure of VSD through a 3-port entry was safe and effective. (J Thorac Cardiovasc Surg 2014;147:863-8)

Ventricular septal defect (VSD) is one of the most common congenital cardiac defects found in children. Minimally invasive surgical approaches have been applied to repair VSDs to minimize surgical trauma and improve the cosmetic results. ${ }^{1,2}$ Totally endoscopic closure of congenital heart defects is an emerging technique whereby the defects are closed entirely by thoracoscopy via small port accesses in the right chest wall. It has been mainly used in the closure of atrial septal defects. $^{3-10}$ Compared with conventional sternotomy, totally thoracoscopic atrial septal defect closure was associated with faster recovery of physical function and a

\footnotetext{
From the Department of Cardiac Surgery, ${ }^{a}$ Qilu Hospital, Shandong University, Jinan, China; School of Biomedical Sciences, ${ }^{b}$ Charles Sturt University, Wagga Wagga, New South Wales, Australia; and Department of Cardiac Surgery, ${ }^{\mathrm{c}}$ Liaocheng People's Hospital and Liaocheng Clinical School of Taishan Medical University, Liaocheng, Shandong, China.

Disclosures: Authors have nothing to disclose with regard to commercial support.

Read at the 93rd Annual Meeting of The American Association for Thoracic Surgery, Minneapolis, Minnesota, May 4-8, 2013.

Received for publication June 9, 2013; revisions received Aug 31, 2013; accepted for publication Oct 27, 2013; available ahead of print Dec 9, 2013.

Address for reprints: Le-Xin Wang, MD, PhD, School of Biomedical Sciences, Charles Sturt University, Wagga Wagga, NSW 2678, Australia (E-mail: 1wang@ csu.edu.au) Or: Zeng-Shan Ma, MD, Department of Cardiac Surgery, Qilu Hospital of Shandong University, Jinan, 252000, China (E-mail: mazengshan31@163.com). $0022-5223 / \$ 36.00$

Copyright (C) 2014 by The American Association for Thoracic Surgery

http://dx.doi.org/10.1016/j.jtcvs.2013.10.065
}

better quality of life. ${ }^{6,7}$ Most previously reported thoracoscopic closures of congenital heart disease required the assistance of robotic techniques. ${ }^{3-11}$ In recent years, we have developed a totally endoscopic technique for the closure of atrial septal defects or VSDs in adults and small children, without the need for robotic assistance. ${ }^{12,13}$ The aim of the present study was to summarize the technical details of the procedure and the clinical outcomes of the patients who underwent totally thoracoscopic repair of a VSD through a 3-port entry without the aid of a robotically assisted surgical system.

\section{PATIENTS AND METHODS \\ Patient Selection}

This study was approved by the institutional review board of Qilu Hospital and Liaocheng People's Hospital. Written informed consent was obtained from all participants before the study. Between June 2009 and March 2013, patients with congenital VSD were selected from the Departments of Cardiac Surgery in the 2 hospitals. The selection criteria were (1) VSD of any size; (2) more than 2 years old with a body weight of more than $15 \mathrm{~kg}$; (3) pulmonary arterial systolic pressure (measured by echocardiography) less than $90 \mathrm{~mm} \mathrm{Hg}$; (4) no previous history of lung disease or surgeries on the right chest; and (5) no other cardiovascular disease or chronic illnesses.

\section{Anesthesia \\ After induction of general anesthesia, a left-sided double-lumen endotracheal tube $(\geq 50 \mathrm{~kg})$ or single-lumen endotracheal tube $(<50 \mathrm{~kg})$ was placed to allow for single-lung ventilation. The respiration rate was}




\section{Abbreviations and Acronyms \\ ICU $=$ intensive care unit \\ $\mathrm{VSD}=$ ventricular septal defect}

set between 18 and 30/min and the arterial oxygen saturation rate was maintained at more than $97 \%$. After the induction of general anesthesia, a transesophageal echocardiography probe was inserted to monitor the VSD closure. Cardiopulmonary bypass was established peripherally through the femoral vein and femoral arteries. ${ }^{14}$ A 24F/29F Carpentier double-lumen catheter (Medtronic Inc, Minneapolis, Minn) or a 14F/22F double-lumen catheter (Kangxin, MN, Changzhou, China) was inserted through the right femoral vein into the inferior and superior vena cava. A second catheter ( $7 \mathrm{~F}$ to $21 \mathrm{~F}$, Medtronic or Kangxin) was inserted into the right femoral artery and was retrogradely advanced to the abdominal aorta for perfusion.

\section{Surgical Techniques}

The patient was positioned in a $15^{\circ}$ to $20^{\circ}$ left lateral decubitus position. Three small incisions (ports) were made on the right side of the chest (Figure 1). Port 1 (1-1.5 cm) was located in the third intercostal space inside the right midclavicular line (Figure 1). This port was for the insertion of surgical instruments such as tissue forceps using the left hand (for a right-handed operator). Port $2(1-1.5 \mathrm{~cm})$ was for the entry of instruments, such as scissors, suture needle, handled by the right hand of the operator. It was located in the fifth or sixth intercostal space outside the right midclavicular line (Figure 1). Port $3(1.5-2.0 \mathrm{~cm})$ was located in the fourth intercostal space between the midaxilliary line and anterior axillary line (Figure 1). This port was for the placement of the thoracoscope.

Once the ports were made, a tissue retractor (Hangtian Kadi, Beijing, China) was inserted into each of the 3 ports to keep the ports open and to facilitate the insertion or withdrawal of the instruments or thoracoscope (Figure 2). First, a thoracoscope (Olympus Corporation, Tokyo, Japan) was inserted through port 3 to visualize the right mediastinum. Under the vision of the thoracoscope, other surgical instruments were inserted through port 1 and port 2 to avoid injuries to the right internal thoracic artery and other vessels inside the right chest during the procedures. Other surgical instruments such as forceps or scissors were conventional instruments for thoracoscopic surgery (Scanlan International, St Paul, Minn).

Pericardiotomy was performed and 1 suture was inserted to suspend the pericardium. Caval snares were placed in the superior and inferior vena cava before commencing cardiopulmonary bypass. A 3-mm incision was made between the left superior pulmonary vein and the left atrium for the insertion of a left atrium drainage tube. The thoracoscope was then removed from port 1 and repositioned through port 2 to visualize the root of the aorta. An aortic crossclamp was positioned on the ascending aorta, and a perfusion needle was inserted through port 3 to the aortic root for the delivery of cold St Thomas' Hospital cardioplegic solution to achieve cardiac arrest. The thoracoscopy was then repositioned through port 3 to visualize the right atrium. Tissue forceps and scissors were inserted through port 1 and port 2 , respectively. After the right atrium was opened from a site parallel to the atrioventricular annulus, 3 stay sutures were placed on the incision to expose the intraatrial structure. The tricuspid valve leaflets were pulled apart and 1 stay suture was placed on the interventricular septum to expose the VSD. For a defect less than $6 \mathrm{~mm}$, direct suture (5-0 or 4-0 Prolene) was used for closure. A bovine patch (Bairen Med Pharma Co, Beijing, China) was used to repair defects of $6 \mathrm{~mm}$ or more (Figure 3), using running Prolene sutures (Ethicon, Somerville, $\mathrm{NJ}$ ). The DeVega technique or an artificial tricuspid valve ring (Bairen Med Pharma Co, Beijing, China) was used to repair tricuspid valve regurgitation.
After VSD closure, the right atrium was closed and the air was exhausted from the left heart. The aortic crossclamp was released and the patient was rewarmed. The integrity of the VSD closure was confirmed by transesophageal echocardiography and protamine sulfate $(1: 1)$ was administered to reverse the actions of heparin. After adequate hemostasis was achieved, all instruments were removed from the chest, and an $18-24 \mathrm{~F}$ chest tube was inserted in the right pleural space through port 2 for drainage. The cannulas in the right femoral vein and artery were removed and incisions on the blood vessels were closed with sutures.

To compare operational data and the requirement for postoperative analgesics between conventional and thoracoscopic VSD closure, 35 patients undergoing open-chest surgery for VSD closure between June 2009 and March 2012 were selected. These patients were matched according to the type of VSD with the last 35 patients in the thoracoscopic group.

\section{Perioperative Management}

Before the surgery, education and counseling were provided to all participants on surgical techniques, possible clinical outcomes, potential complications, and postoperative self-care measures. Lung function tests were routinely performed in all patients before the surgery. The lungs were inflated every 20 minutes during the operation. The femoral artery and vein were assessed by color Doppler ultrasonography. Bedside chest radiographs were routinely obtained in the intensive care unit (ICU) to exclude complications in the lungs. Mechanical ventilation was ceased once the patient's hemodynamics stabilized in the operating room or in the ICU.

\section{Statistical Analysis}

SPSS v13.0 (SPSS, Inc, Chicago, Ill) was used for the statistical analysis. Quantitative variables are expressed as the mean \pm standard deviation. Differences between multiple groups were compared by one-way analysis of variance. Categorical variables were compared by the $\chi^{2}$ test.

\section{RESULTS}

A total of 276 patients were screened initially and 119 were recruited to this study. The main reasons for exclusion were (1) less than 2 years old or body weight less than $15 \mathrm{~kg}$ $(\mathrm{n}=122)$; (2) older than 2 years but, as seen on the Doppler ultrasonography examination, the femoral artery or vein was too small $(<1.3 \mathrm{~mm}$ in diameter $)$ for canalization $(\mathrm{n}=11)$; (3) pulmonary artery systolic pressure $90 \mathrm{~mm}$ $\mathrm{Hg}$ or higher ( $\mathrm{n}=17)$; and (4) a history of lung disease or right chest surgery preventing insertion of the thoracoscope $(\mathrm{n}=5)$.

Of the 119 patients selected, 66 were boys and 53 were girls; the mean age was $7.1 \pm 3.6$ years (range, 2-36 years). The mean body weight was $32.4 \pm 7.8 \mathrm{~kg}$ (range, $18-68 \mathrm{~kg}$ ). Echocardiographic examination showed type I (membranous) VSD in 64, type II (paramembranous) VSD in 51, type III (arteriovenous canal type or inlet septum type) in 3 , and type IV in 1 . The size of the defect ranged from 4 $\mathrm{mm}$ to $14 \mathrm{~mm}$. Twenty-eight patients had mild pulmonary hypertension (pulmonary arterial pressure, $30-40 \mathrm{~mm}$ $\mathrm{Hg}), 22$ patients had moderate pulmonary hypertension (pulmonary arterial pressure, $40-59 \mathrm{~mm} \mathrm{Hg}$ ), and 6 had severe pulmonary hypertension (pulmonary arterial pressure, $60-90 \mathrm{~mm} \mathrm{Hg}$ ). Sixteen patients also had tricuspid regurgitation as a result of right ventricular dilatation. 


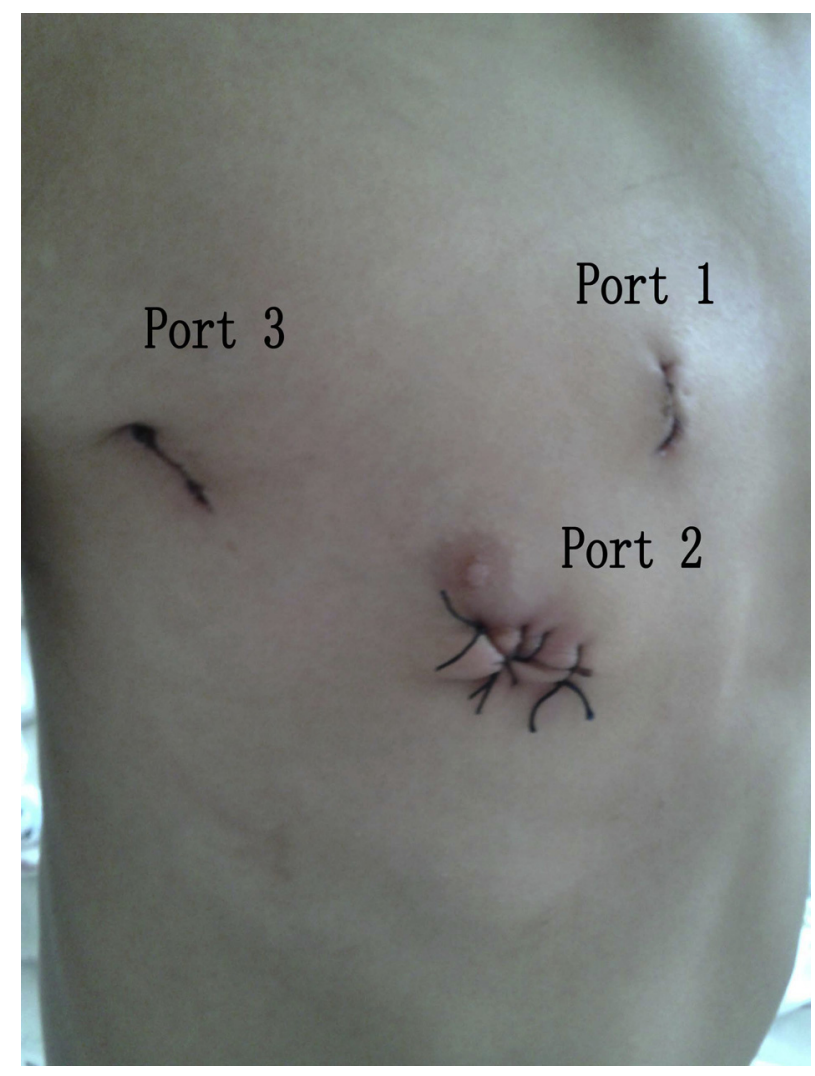

FIGURE 1. Location of the 3 ports on the right chest wall.

Valvuloplasty using the DeVega technique and Edward ring was performed in 9 and 3 patients, respectively, after the VSD repair in the same procedure. Follow-up echocardiographic studies showed no tricuspid regurgitation in these patients.

One patient had bleeding as a result of injury to the inferior vena cava and the right atrium. Another patient had bleeding from the ascending aortic where a needle was inserted to perfuse cardioplegic solution. In both cases, the

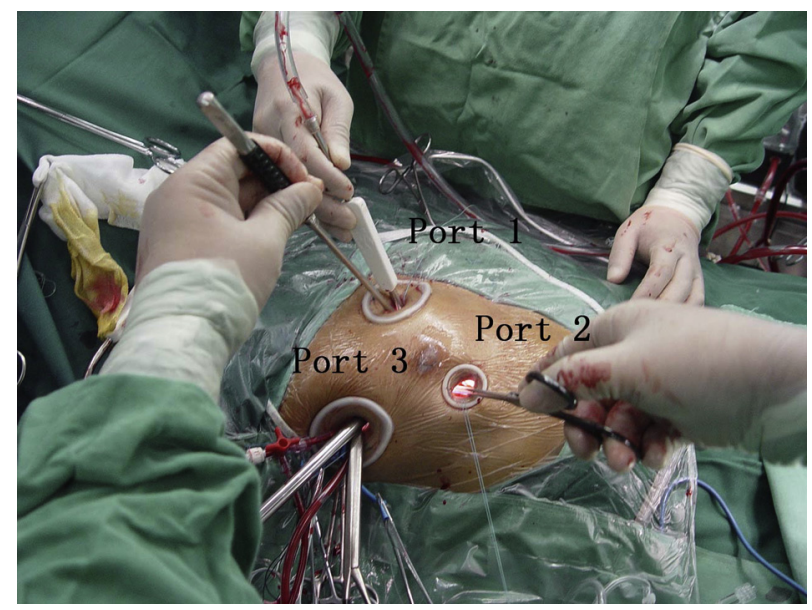

FIGURE 2. Function of the 3 ports.

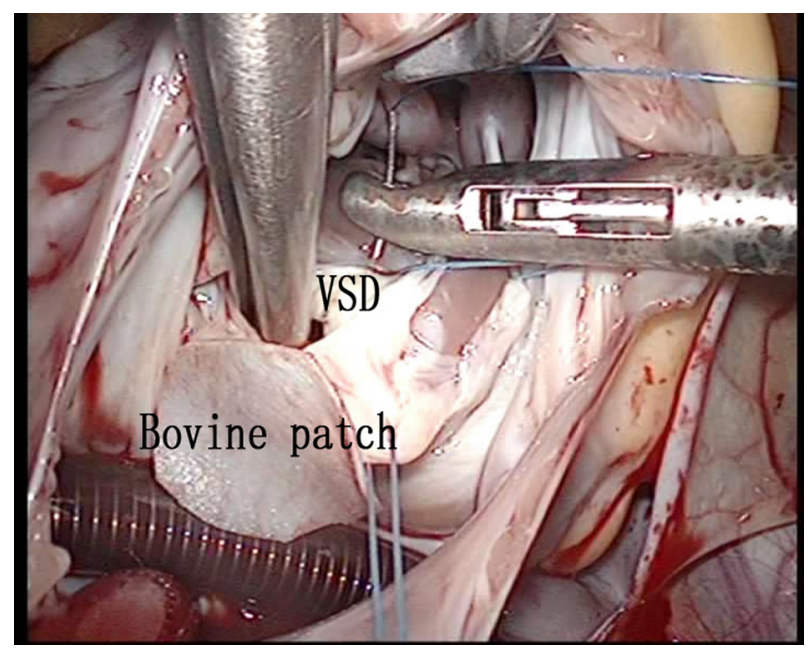

FIGURE 3. Closure of a ventricular septal defect (VSD).

chest incision at port 2 was extended to $4-5 \mathrm{~cm}$ for better visualization of the bleeding sites, which were successfully stopped with sutures. Thoracoscopic VSD repair was completed successfully after the bleeding from the inferior vena cava or ascending aorta was stopped.

All patients survived the operation and were discharged from the hospital in 4 to 6 days. No patient in this cohort underwent reoperation for bleeding after the VSD repair. The mean cardiopulmonary bypass time was $42.2 \pm 9.8$ minutes (range, 34-142 minutes). The mean aortic crossclamp time was $32.5 \pm 7.3$ minutes (range, 25-57 minutes). Once the aortic clamp was removed, the heart beat resumed spontaneously in 84 patients. In 33 patients, a 50-W direct current shock through the chest wall was administered to cardiovert the fibrillating heart back to sinus rhythm. The mean mechanical ventilation time was $2.1 \pm 1.2$ hours (range, 1-19 hours) and the mean time in the ICU was $11.2 \pm 3.6$ hours (range, 4-28 hours). There was no statistically significant difference in the mean mechanical ventilation time ( $2.2 \pm$ 1.3 vs $2.0 \pm 1.1$ hours, $P>.05)$ and time in the ICU $(11.4 \pm 3.9$ vs $11.0 \pm 3.2$ hours, $P>.05)$ between the 6 patients with severe preoperative pulmonary hypertension and those with mild or moderate preoperative pulmonary hypertension.

In all patients, transesophageal echocardiography immediately after the VSD repair showed complete closure with no residual shunt. Three patients $(2.26 \%)$ experienced arrhythmia, 1 had high-degree atrioventricular conduction block, which recovered spontaneously within 24 hours of the operation. One patient had third-degree atrioventricular conduction block that had not recovered spontaneously within 7 days of the operation and was fitted with a permanence pacemaker. The other patient had atrial fibrillation after the VSD repair but sinus rhythm returned after 52 hours. One $(0.75 \%)$ had residual shunting measuring $1 \mathrm{~mm}$, which closed itself 1 month after the operation. One patient 
TABLE 1. Comparison of operational data between the thoracoscopic closure group (study) and the control group

\begin{tabular}{lccc}
\hline & $\begin{array}{c}\text { Study } \\
(\mathbf{n}=\mathbf{1 1 9})\end{array}$ & $\begin{array}{c}\text { Control } \\
(\mathbf{n}=\mathbf{3 5})\end{array}$ & $\boldsymbol{P}$ \\
\hline Age (y) & $7.1 \pm 3.6$ & $7.8 \pm 4.3$ & .106 \\
Male, n (\%) & $66(55.4)$ & $18(51.4)$ & .623 \\
Body weight (kg) & $32.4 \pm 7.8$ & $34.5 \pm 9.2$ & .189 \\
Perimembranous VSD, n (\%) & $55(46.2)$ & $16(45.7)$ & .789 \\
Membranous VSD, n (\%) & $64(53.8)$ & $19(55.3)$ & .671 \\
Total operation time (min) & $86 \pm 15$ & $124 \pm 18$ & .002 \\
Cardiopulmonary bypass (min) & $42.2 \pm 9.8$ & $48 \pm 4$ & .077 \\
Aortic crossclamp time (min) & $32.5 \pm 7.3$ & $29.9 \pm 3.4$ & .059 \\
Mechanical ventilation time (h) & $0.3 \pm 0.4$ & $4.9 \pm 3.2$ & .006 \\
Intensive care stay (h) & $11.0 \pm 2.6$ & $22.9 \pm 4.7$ & .009 \\
Postoperative hospital stay (d) & $4.2 \pm 1.1$ & $6.6 \pm 2.1$ & .036 \\
Volume of chest drainage (mL) & $55 \pm 12$ & $237 \pm 66$ & .001 \\
Rate of blood transfusion, n (\%) & $21(17.6)$ & $23(65.7)$ & .001 \\
Postoperative analgesics, n (\%) & $38(31.9)$ & $26(74.3)$ & .003 \\
\hline
\end{tabular}

$(0.75 \%)$ had right pulmonary atelectasis, which recovered in 2 days after chest physiology therapy.

Twenty-one patients $(16 \%)$ required a blood transfusion of $400 \mathrm{~mL}$ each during the procedure. The total volume of the postoperative chest drain ranged from 30 to $460 \mathrm{~mL}$ (mean, $55 \pm 12 \mathrm{~mL}$ ). Patients have been followed up for $5.2 \pm 3.9$ months (3-12 months) with no signs of residual shunt on transthoracic echocardiography. Patients resumed work or schooling between 14 and 23 days (median, 16 days) after the surgery.

As shown in Table 1, there was no significant difference in age, sex, cardiopulmonary bypass time, and aortic crossclamp time between the thoracoscopic and control groups $(P>.05)$. In the thoracoscopic group, total operation time, mechanical ventilation time, and blood loss during surgery were significantly less than in the control group $(P<.01)$, and the ICU or hospital stays were shorter $(P<.05)$. In the thoracoscopic group, $38(31.9 \%)$ patients required opioid analgesics. In the control group, 26 patients $(74.2 \%, P<.01)$ were administered with opioid analgesics for 1 to 2 days after the surgery.

\section{Comment}

We have previously reported our initial experience on totally thoracoscopic repair of VSD in 36 patients from a single hospital. ${ }^{12}$ The present study is an extension of our previous report and summarizes the results of 119 patients treated by the same operator (Ma ZS) at 2 tertiary hospitals. Compared with patients treated through conventional sternotomy, the totally thoracoscopic approach was associated with a shorter operation time, shorter postoperative hospital stay and shorter time in the ICU, lower rate of blood transfusion, and lower rate of postoperative analgesics. A permanent complication was observed in 1 patient who had complete atrioventricular conduction block that required insertion of a permanent pacemaker. There were no other long-term complications during a mean follow-up of 5.2 months.

In our first report, we excluded patients with supracristal or muscular VSD because of the difficulties in visualizing and accessing these types of defects. ${ }^{12}$ In the last 2 years, we have extended totally thoracoscopic closure to almost all types of VSDs. Suprcristal or subarterial VSDs with a diameter less than $4 \mathrm{~mm}$ can be closed by direct suturing without the need for a patch. Theoretically, muscular VSDs (type IV) close to the apex or distant to the tricuspid annulus are difficult to access through the chest ports. Fortunately, this type of VSD is rare and, in the present cohort, we encountered only 1 patient with muscular VSD, which was successfully closed with the totally thoracoscopic approach.

Totally thoracoscopic closure of VSD as well as other types of congenital heart defects can be limited by the patient's age or body weight. Younger children or children with a low body weight tend to have smaller femoral veins and arteries, which may hinder the insertion of catheters for peripheral cardiopulmonary bypass. Also, these patients often have a thoracic cavity that is too small to allow optimal manipulation of the thoracoscope and other surgical devices within the chest. In the present study, 276 patients were initially screened for total thoracoscopic closure but almost half of these patients were excluded mostly because they were less than 2 years old or their body weight was less than $15 \mathrm{~kg}$. Some patients were older than 2 years but preoperational Doppler ultrasonography examination showed a small femoral artery or vein that was not suitable for catheterization. In younger children with a small femoral vein $(<1.3 \mathrm{~mm}$ in diameter), peripheral cardiopulmonary bypass can be established through catheterization of the internal carotid vein to enable totally thoracoscopic closure of VSD. ${ }^{13}$ Overall, there is limited experience in totally thoracoscopic VSD repair in younger children with small femoral blood vessels and a small chest cavity, and conventional closure via sternotomy is probably a safer option.

In the present study, we also included 6 patients with a preoperational pulmonary systolic pressure between 60 and $90 \mathrm{~mm} \mathrm{Hg}$; in the past, these patients were excluded. ${ }^{12}$ There were no perioperative mortalities or short-term complications in these patients with severe preoperational pulmonary hypertension. There was no statistically significant difference in the mean mechanical ventilation time and duration of stay in the ICU between the 6 patients with severe preoperative pulmonary hypertension and those with mild or moderate preoperative pulmonary hypertension. However, the short- and long-term safety of patients with severe preoperational pulmonary hypertension after totally thoracoscopic VSD repair needs further investigation through larger and multicentered trials.

In this study, we were unable to conduct a randomized trial to compare the safety and efficacy of totally endoscopic 
and conventional surgery for VSD repair. In addition, the investigators were blinded to the postoperative care of the patients. Therefore, interpretation of the postoperative mechanical ventilation time, ICU and hospital stay, and the use of postoperative analgesics between the 2 groups requires some caution. In our center, postoperative extubation is usually performed in the ICU because many patients ( $47 \%$ in the present study) have pulmonary hypertension when presented to us for corrective surgery as a result of late diagnosis. With the endoscopic approach for VSD closure, we have become more confident about extubating in the operating rooms, hence the reduced mechanical ventilation time and shorter ICU and hospital stays. Sixtysix percent of control patients required blood transfusion as a result of blood loss from the mediastinum and the sternotomy wound during the operation, whereas the transfusion rate in the study group was only $18 \%$.

In the past, robotics techniques have been used for totally endoscopic repair of congenital heart defects, mostly atrial septal defects. ${ }^{3-6}$ In the present study, robotics was not used, and the addition of these robotics techniques probably would not further enhance our techniques in VSD repair. With the current endoscopic approach, we were able to display the intrathoracic operation field on a large television screen and the operators were able to visualize the thoracic and cardiac anatomy and the intrathoracic surgical instruments very much in the same fashion as in an open-chest surgery. The technique is also relatively simple to learn. ${ }^{8,12}$

In conclusion, totally thoracoscopic repair of VSDs through a 3-port entry without the aid of a robotically assisted surgical system seems to be safe and effective. However, this technique is only suitable for children who are 2 years or older with a body weight of more than $15 \mathrm{~kg}$.

\section{References}

1. Penny DJ, Vick GW III. Ventricular septal defect. Lancet. 2011;377:1103-12.

2. Doll N, Walther T, Falk V, Binner C, Bucerius J, Borger MA, et al. Secundum ASD closure using a right lateral minithoracotomy five-year experience in 122 patients. Ann Thorac Surg. 2003;75:1527-31.

3. Argenziano M, Oz MC, Kohmoto T, Morgan J, Dimitui J, Mongero L, et al. Totally endoscopic atrial septal defect repair with robotic assistance. Circulation. 2003;108(Suppl 2):191-4.

4. Bonaros N, Schachner T, Oehlinger A, Ruetzler E, Kolbitsch C, Dichtl W, et al. Robotically assisted totally endoscopic atrial septal defect repair: insights from operative times, learning curves, and clinical outcome. Ann Thorac Surg. 2006;82:687-93.

5. Wimmer-Greinecker G, Dogan S, Aybek T, Khan MF, Mierdl S, Byhahn C, et al. Totally endoscopic atrial septal repair in adults with computer-enhanced telemanipulation. J Thorac Cardiovasc Surg. 2003;126:465-8.

6. Morgan JA, Peacock JC, Kohmoto T, Garrido MJ, Schanzer BM, Kherani AR, et al. Robotic techniques improve quality of life in patients undergoing atrial septal defect repair. Ann Thorac Surg. 2004;77:1328-33.

7. Ma ZS, Yin QY, Dong MF, Feng ZY, Wang LX. Quality of life in patients undergoing totally thoracoscopic closure for atrial septal defect. Ann Thorac Surg. 2011;92:2230-4.

8. Ma ZS, Dong MF, Yin QY, Feng ZY, Wang LX. Totally thoracoscopic repair of atrial septal defect without robotic assistance: a single-center experience. J Thorac Cardiovasc Surg. 2011;141:1380-3.
9. Ma ZS, Dong MF, Yin QY, Feng ZY, Wang LX. Totally thoracoscopic closure for atrial septal defect on perfused beating hearts. Eur J Cardiothorac Surg. 2012;41: 1316-9.

10. Liu G, Qiao Y, Zou C, Ma L, Ni L, Zeng S, et al. Totally thoracoscopic surgical treatment for atrial septal defect: mid-term follow-up results in 45 consecutive patients. Heart Lung Circ. 2013;22:88-91.

11. Yao DK, Chen H, Ma ZS, Ma LL, Wang LX. Totally endoscopic closure of atrial septal defect- a Meta-analysis and systematic review. Heart Lung Circ. 2013;22: 433-40.

12. Ma ZS, Dong MF, Yin QY, Feng ZY, Wang LX. Totally thoracoscopic repair of ventricular septal defect: a short-term clinical observation on safety and feasibility. J Thorac Cardiovasc Surg. 2011;142:850-4.

13. Ma ZS, Wang JT, Dong MF, Chai SD, Wang LX. Thoracoscopic closure of ventricular septal defect in young children: technical challenges and solutions. Eur J Cardiothorac Surg. 2012;42:976-9.

14. Zhang ZW, Zhang XJ, Li CY, Ma LL, Wang LX. Technical aspects of anesthesia and cardiopulmonary bypass in patients undergoing totally thoracoscopic cardiac surgery. J Cardiothorac Vasc Anesth. 2012;26:270-3.

\section{Discussion}

Dr William M. DeCampli (Orlando, Fla). In this series of 119 patients undergoing endoscopic VSD repair Professor Ma and colleagues have demonstrated the safety and efficacy of the procedure and the superiority of a number of outcome measures compared with the conventional open technique. This group's results build on their 3 previously published series of endoscopic ASD and VSD repairs in our journals.

For those who have not embraced lesser invasive surgery, it is no longer just video gaming. Prospective randomized studies now demonstrate its superiority in a broad variety of operations in multiple surgical specialties. Although there is no class 1 evidence for better outcomes in pediatric cardiac surgery, case-control studies such as this one support that view. In operations with essentially zero mortality, outcome measures such as length of stay and time to return to school or to work are important and do seem to be better with a lesser invasive approach.

I have 3 questions and I'll give them to you one at a time.

First, you report significantly less bleeding and transfusion requirement, $16 \%$ versus $67 \%$, with the endoscopic versus conventional approach. That's a large difference. To what do you ascribe this difference? The cardiopulmonary bypass times are comparable. Is all the blood loss from the skin and the sternal incision?

Dr Ma. Yes, I think that we may ascribe the blood loss to the incision and partly the sternotomy. The average body weight of children is much smaller than the adult population. The blood loss during this period was comparatively larger than in adults.

Dr DeCampli. My second question is as follows. Many groups, including one of our moderators, have published favorable results of a sternum-sparing subxiphoid approach for repair not only of VSD but also tetralogy of Fallot, arteriovenous canal and even mitral valve repair in children. This approach avoids size-limiting femoral cannulation and is more versatile. What are your thoughts on comparing this approach with your totally endoscopic approach?

Dr Ma. I don't have experience in that procedure, but it has limitations in many operations. I think our procedures leads to less trauma, and has better cosmetic results.

Dr DeCampli. Lastly, why cannot you repair muscular VSDs with your approach? How about inlet VSDs? Granted that robots 
are expensive, but do you think a robotic approach could nonetheless potentially increase your versatility?

Dr Ma. I think that muscular VSDs are usually situated near the apex and usually have many outlets on the right ventricular side. So I have never tried to do this.
The second, I have done many inlet VSD repairs. I think that an inlet VSD is one of the best indications for totally thorascopic procedures, whether the repair is done by direct sutures or patches. Thirdly, I don't have experience in robotic procedures because my hospital does not have this equipment. 